\title{
Autoimmunhepatitis
}

\section{Gut verträgliche Steroidtherapie}

- Die Autoimmunhepatitis (AlH) ist eine seltene Lebererkrankung, die unbehandelt zur Leberzirrhose und zum akuten Leberversagen führen kann. Das klinische Bild der Erkrankung sei sehr unterschiedlich, führte PD Dr. Elmar Jäckel, Hannover, aus. Es reiche von asymptomatischen minimalen Erhöhungen der Transaminasen bis hin zum fulminanten Leberversagen. Die Diagnose basiert auf einer selektive Erhöhung des IgG, dem Nachweis von Auto-Antikörpern, dem fehlenden Nachweis einer Virushepatitis und dem histologischen Nachweis einer Hepatitis, wobei letzterer unverzichtbar ist.

\section{Wenige Nebenwirkungen}

Die Erkrankung lässt sich therapeutisch gut beeinflussen. Mittel der Wahl für die Induktion der Remission sind Kortikosteroide, für die Remissionserhaltung empfiehlt sich die Gabe von Azathioprin. Die Steroide sollten anfänglich ausreichend hoch dosiert eingesetzt werden. Bei Prednisolon empfiehlt sich eine Dosierung von $1 \mathrm{mg} / \mathrm{kg} \mathrm{KG}$. Bei gutem Ansprechen kann die Dosis schnell reduziert werden.

Doch die hochdosierte Gabe von Prednisolon ist mit unangenehmen Nebenwirkungen assoziiert. Deshalb empfiehlt sich als verträglichere Alternative das topische Steroid Budesonid (Budenofalk ${ }^{\oplus}$ ) - es sei denn, es läge bereits eine Leberszirrhose vor. Diese Substanz hat nur eine geringe systemische Bioverfügbarkeit, sodass steroidtypische Nebenwirkungen sehr viel seltener sind. In einer randomisierten Studie zeigte sich, dass die Gabe von $3 \times 3$ mg Budesonid als Induktionsdosis Prednisolon keinesfalls unterlegen ist.

Dr. Peter Stiefelhagen .

- Quelle: 19. Symposium „Aktuelle Hepatologie 2013“ im Rahmen des 119. Kongresses der Deutschen Gesellschaft für Innere Medizin; Wiesbaden, April 2013; (Veranstalter: Falk)

\section{Medizinische Thromboseprophylaxestrümpfe}

\section{Ungeschmälerte Bedeutung}

\section{- Medizinische Thromboseprophylaxe-} strümpfe (MTPS) spielen nach wie vor eine wichtige Rolle in der Thromboseprophyla$x e$, betont Prof. Knut Kröger, Angiologe aus Krefeld. MTPS sind seit mehr als 40 Jahren auf dem Markt. Aber nicht alle Fabrikate sind in dieser Zeit konsequent weiterentwickelt worden, bedauerte Dr. Ulrich Wegener, Ingenieur der TU Berlin. Dies sei bei einem Druckverlaufstest deutlich geworden, den die TU Berlin nach der HoSy-Methode durchgeführt hat. „Strumpf ist nicht gleich

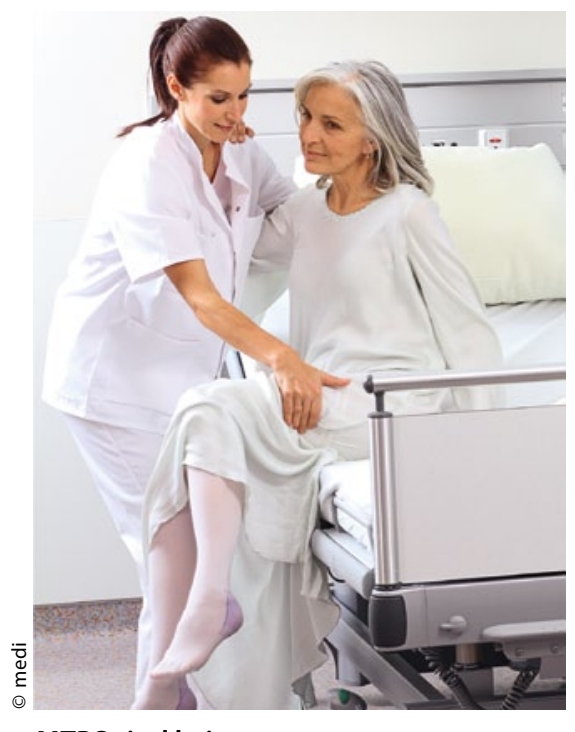

MTPS sind keineswegs out ...
Strumpf", so Prof. Marc Kraft, TU Berlin, und verriet erste Ergebnisse: „Testsieger ist der MTPS der Firma medi. Nur dieser Strumpf konnte in unserem Test vollumfänglich überzeugen."

Zur Klärung der Evidenz von MTPS kündigten Kröger und Kraft weitere Untersuchungen an. „Wir haben bereits Ultraschalltests gemacht, um die Fließgeschwindigkeit des Blutes in den Gefäßen verschiedener Patienten mit und ohne Strumpf zu messen. Die Ergebnisse bestätigen die physikalische Wirkweise der Strümpfe." MRT-Aufnahmen sollen weitere Erkenntnisse bringen.

\section{Schadensersatzklage droht}

Juristen warnen davor, den Einsatz von MTPS einzustellen und nur noch medikamentös zu behandeln. In Potsdam sei bereits eine Klinik verklagt worden, so die Juristen Prof. Volker Großkopf und Dr. Hubert Klein, beide aus Köln. Gesundheitsökonom Prof. Wirlfried von Eiff, Münster, pflichtete bei: „Das wäre Sparen an der falschen Stelle. MTPS sind ein vergleichsweise kostengünstiges Mittel zur Vermeidung von Thrombosen. Das konnten wir bei unseren Untersuchungen nachweisen."

Red. .

- Nach Informationen von MDI; 15. Bremer Pflegekongress, Bremen, Mai 2013
Kurz notiert

Wenn im Sommer die Nase läuft $>$ Rund jeder fünfte akute Infekt der oberen Luftwege ereignet sich im Sommer. Typische Ursachen sind Unterkühlungen durch Zugluft oder Klimaanlagen, Auskühlen beim Wassersport sowie ein ungenügender Druckausgleich auf den Flügen in den Urlaub, führte Prof. Hans Behrbohm, Berlin, aus. GeloMyrtol ${ }^{\circledast}$ forte, ein Destilat aus einer Mischung der rektifizierten Öle von Eukalyptus, Süßorange, Myrte und Zitrone, wirkt gut gegen eine katarrhalische Sinusitis, da es das zähe Sekret der Atemwege löst, das Bakterienwachstum hemmt, schädliche freie Radikale einfängt und Leukotriene bremst.

Auch gegen die allergischen Rhinosinusitiden im Sommer empfiehlt sich laut Behrbohm neben der üblichen Behandlung mit Antihistaminika eine zusätzliche Therapie mit GeloMyrtol $^{\circledR}$ forte. Das Destillat beschleunigt nämlich auch die mukoziliäre Clearance: Allergene auf dem Sekretfilm der Schleimhaut werden so schneller abgeschwemmt und verschluckt.

Red. .

- Quelle: Expertengespräch „Neue Trends in der Sinusitis-Therapie", Hamburg, April 2013 (Veranstalter:Pohl-Boskamp) 